\title{
Vitamin Supplementation as Possible Prophylactic Treatment against Migraine with Aura and Menstrual Migraine
}

\author{
Munvar Miya Shaik and Siew Hua Gan \\ Human Genome Centre, School of Medical Sciences, Universiti Sains Malaysia, 16150 Kubang Kerian, Kelantan, Malaysia \\ Correspondence should be addressed to Siew Hua Gan; shgan@usm.my
}

Received 24 December 2014; Accepted 17 February 2015

Academic Editor: Abdel A. Abdel-Rahman

Copyright ( 2015 M. M. Shaik and S. H. Gan. This is an open access article distributed under the Creative Commons Attribution License, which permits unrestricted use, distribution, and reproduction in any medium, provided the original work is properly cited.

\begin{abstract}
Migraine is the most common form of headache disorder globally. The etiology of migraine is multifactorial, with genetic components and environmental interactions considered to be the main causal factors. Some researchers postulate that deficits in mitochondrial energy reserves can cause migraine or an increase in homocysteine levels can lead to migraine attacks; therefore, vitamins could play a vital role in migraine prevention. For instance, riboflavin influences mitochondrial dysfunction and prevents migraine. Genes such as flavoenzyme 5,10-methylenetetrahydrofolate reductase (MTHFR), especially the C677T variant, have been associated with elevated plasma levels of homocysteine and migraine with aura. Homocysteine catalyzation requires the presence of vitamins $B_{6}, B_{12}$, and folic acid, which can decrease the severity of migraine with aura, making these vitamins potentially useful prophylactic agents for treating migraine with aura. Menstrual migraine, on the other hand, is associated with increased prostaglandin (PG) levels in the endometrium, indicating a role for vitamin E, which is an anti-PG. Vitamin C can also be used as a scavenger of reactive oxygen species for treating neurogenic inflammation in migraine patients. This paper reviews possible therapies based on vitamin supplementation for migraine prophylaxis, focusing on migraine with aura and menstrual migraine.
\end{abstract}

\section{Introduction}

Migraine is a common, painful, and disabling condition characterized by recurrent, unilateral, and pulsatile attacks of headache that can be moderate to severe in intensity [1]. Most migraine attacks begin at puberty and affect those aged between 35 and 45 years. The prevalence of migraine is higher in women (5\% to $25 \%)$ than in men $(2 \%$ to $10 \%)[2,3]$. The incidence of migraine attacks tends to peak in the middleaged group but is reported to be lower among adolescents and the elderly (aged above 60 years) [3]. Migraine symptoms vary among individuals, and different symptoms may present during different attacks. Migraine attacks may also differ in length and frequency, usually lasting from 4 to 72 hours. Although individuals may be free of symptoms between attacks, the attacks have an enormous impact on work, family, and social lives.

There are several types of migraines, and they are diagnosed based on the symptoms experienced. Some are triggered by seasonal changes and others by alterations in hormone levels, especially in women. In 1988, the International Headache Society produced a system for classifying migraines, a system also adopted by the World Health Organization [4]. This system was updated in January 2004 [5] and is the established basis for defining types of headaches. The most common forms of migraine fall into two main categories, migraine with aura (MA) and migraine without aura (MO). MO is the most common type of migraine, and approximately $80 \%$ of migraine patients are reported to have this type [3]. MA is primarily characterized by the presence of focal neurological symptoms such as blurred vision, vertigo, or hallucination, and these symptoms usually precede or accompany the headache attacks. Some patients also experience a premonitory phase, which occurs hours or days before the headache, followed by a resolution phase. Premonitory and resolution phase symptoms including hyperactivity and hypoactivity, depression, cravings for particular foods, repetitive yawning, and other less typical symptoms are reported by some patients. These symptoms differ from migraine without aura, which is not preceded by aura and may last from 4 to 
72 hours. Nearly $31 \%$ of migraine patients are reported to have 3 or more headache attacks per month, with $54 \%$ of migraine patients reported to have severe impairment in daily functions both at home and at work [3]. Similar prevalence rates have been shown in other populations (European and Asian) with a comparable distribution pattern based on age and sex [6].

It was hypothesized that mitochondria may play a role in the pathogenesis of migraine and the roles of mitochondria disorders and migraine have been well established [710]. Mitochondria are small organelles that play a central role in energy generation, reactive oxygen species (ROS) production, regulation of apoptosis, and control of calcium $(\mathrm{Ca} 2+)$ homeostasis [7]. Since mitochondria play a vital role in the primary mechanism of $\mathrm{Ca} 2+$ sequestration in a cell, its dysfunction is postulated to lead to pain hypersensitization [11]. In addition, vasoconstriction during cortical spreading depression (CSD) is caused by increased $\mathrm{Ca} 2+$ concentrations within astrocytes. This process is mediated by phospholipase A2 which is an arachidonic acid metabolite [12]. Mitochondria are crucial for the normal functioning of neurons due to their involvement in $\mathrm{Ca} 2+$ homeostasis. Therefore, an imbalance of $\mathrm{Ca} 2+$ could lead to a range of downstream imbalances, further contributing to increased migraine susceptibility [9].

Cytochrome-c oxidase (COX), also known as complex $\mathrm{IV}$, of the mitochondria respiratory transport chain binds to nitric oxide (NO) [13]. In vessels rich in COX, sequestration of NO may occur, which prevents vasodilatation. COXnegative fibers with increased fat accumulation have also been observed in some patients with migraine [7]. To date, there existed only a single case report of a genetic defect in mitochondria DNA being associated with cluster headache [14]. In addition, two common mitochondria DNA polymorphisms $(16519 \mathrm{C} \rightarrow \mathrm{T}$ and $3010 \mathrm{G} \rightarrow \mathrm{A})$ have been associated with pediatric cyclic vomiting syndrome and migraine [15].

Oxidative stress has been proposed as a relevant event in the pathogenesis of headaches [16]. Oxidative stress was determined by measuring coenzyme Q10, catalase, and lipid peroxidation (LPO) levels. It is believed that oxidative stress and LPO play a role in the pathogenesis of migraine by regulating cerebral blood flow and energy metabolism both of which may constitute a trigger threshold for migraine attacks $[17,18]$. It is known that LPO levels indirectly reflect generation of intracellular ROS as a consequence of oxidative stress as ROS are implicated in pain etiology. Therefore, it is hypothesized that antioxidants (in the form of either vitamins or cofactors) may help to reduce the levels of free radicals which play a vital role in mitochondrial disorders.

Preventive treatment for migraine helps reduce disability by decreasing the frequency, severity, and duration of migraine attacks. The main aim of preventive treatment is to improve the acute treatment response during attacks as well as quality of life (QOL). Preventive treatment is especially crucial for patients with severe migraine attacks and migraine-related disability. Recurring headache-induced disability is a major concern in migraine patients, as it impairs their QOL and ability to work. According to the World Health Organization, migraine is the 19th leading cause of disability in total years lived [6]. Furthermore, migraine is an economic burden to the patient's family as well as their country. In the USA alone, it is estimated that migraine costs approximately USD 1 billion per year in direct medical costs and USD 13 billion per year in lost work productivity [19].

The goal of using a preventive treatment mirrors the criteria for initiating such a treatment. The primary goals of preventive treatment are reduced frequency and severity of migraine headaches, and it is important that these goals be communicated to patients [20-22]. It is also crucial to emphasize early on to patients that migraine has no cure and, therefore, that the goal is to manage the disease and reduce its burden. A patient's disability could be reduced when these main goals are achieved. Other goals of preventive treatment include reducing the use of acute drugs and decreasing visits to the emergency room or the need for surgery. Patients also need to understand that it may take time for a drug to become effective, most often two to three months, even when administered in adequate doses. Asking patients to keep a headache calendar or diary is an effective way to monitor progress.

Medication choices are somewhat limited by their availability in each country. Nonetheless, the first choices of treatment are beta-adrenoceptor blockers (propranolol) [23], calcium channel blockers (flunarizine) [24], anticonvulsants (topiramate) [25], selective serotonin reuptake inhibitors [26], and anticonvulsant drugs (valproic acid) [27]. Betaadrenoceptor blockers are particularly useful in patients also suffering from hypertension or tachycardia. Although topiramate is widely used as a first-line treatment for episodic and chronic migraine, it leads to weight loss and is also associated with adverse cognitive effects. Valproic acid and flunarizine are also considered to have very good prophylactic properties for migraine, yet valproic acid is often associated with adverse effects and flunarizine is unavailable in many countries, including the United States. According to a Cochrane review [26], selective serotonin reuptake inhibitors for migraine treatment are no more efficacious than placebo. The prophylactic properties of other agents such as magnesium, riboflavin, and coenzyme Q10 are low at best, but their lack of severe adverse effects makes them good alternative treatment options [28-30].

Some studies have shown an association of hyperhomocysteinemia with migraine as well as the roles of vitamins $\mathrm{B}_{6}, \mathrm{~B}_{9}$, and $\mathrm{B}_{12}$ in lowering the levels of homocysteine in patients with hyperhomocysteinemia [31-35]. There is also evidence that riboflavin (vitamin $\mathrm{B}_{2}$ ) reduces the frequency of migraine attacks [36]. Vitamins also act as antioxidants and work effectively in oxidative stress to slow down the disease progression. This review highlights the roles of several types of vitamins that have the potential to be used as migraine prophylaxis.

\section{Vitamins}

2.1. Riboflavin (Vitamin $B_{2}$ ). Riboflavin is a nutrient found in milk, eggs, malted barley, liver, kidney, heart, and leafy vegetables, with yeast considered the richest natural source. Studies have investigated the potential role of riboflavin in preventing migraine symptoms [37]. The interictal reduction 


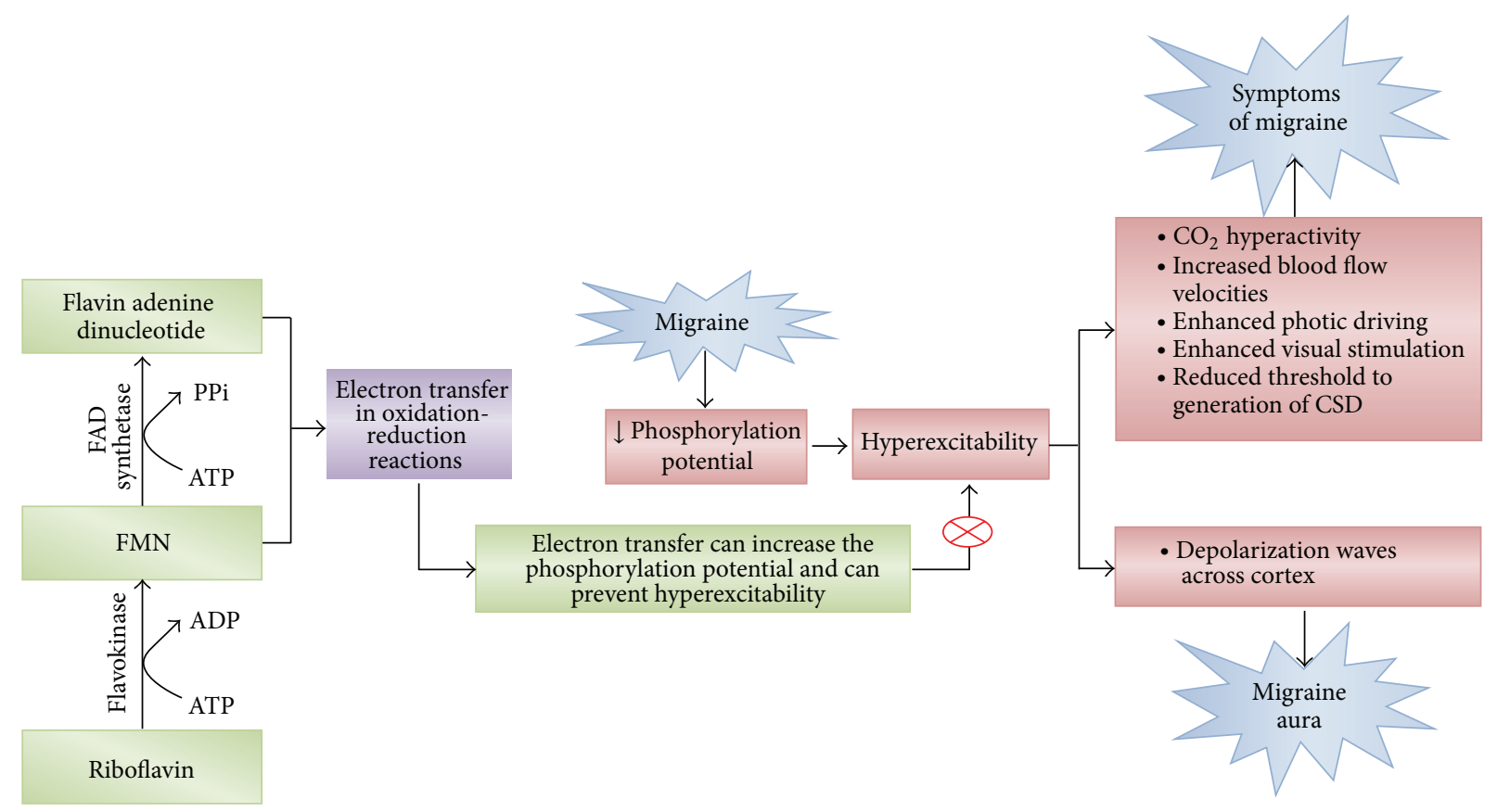

FIGURE 1: Schematic diagram depicting the possible roles of riboflavin in ameliorating migraine. ATP: adenosine triphosphate, ADP: adenosine diphosphate, FAD: flavin adenine dinucleotide, PPi: pyrophosphate (anion $\mathrm{P}_{2} \mathrm{O}_{7}{ }^{4-}$ ), $\mathrm{CO}_{2}$ : carbon dioxide, and CSD: cortical spreading depression. Red circled times symbol: inhibition of the pathway.

of phosphorylation potential in the brain and muscles of migraineurs by riboflavin has previously been reported [38, 39]. The principal forms of riboflavin in tissues and cells are flavin mononucleotide (FMN) and flavin adenine dinucleotide (FAD), which are also coenzymes (Figure 1). These forms of riboflavin are involved in transferring electrons in oxidation-reduction reactions. Patients with mitochondrial encephalomyopathy, lactic acidosis, and stroke-like episodes also show reduced metabolism in mitochondria and experience migraine-like headaches, which have been found to be alleviated by riboflavin [40].

Evidence indicates hyperexcitability in migraine, as shown by carbon dioxide hyperreactivity, elevated cerebral blood flow velocities (observed in transcranial Doppler studies), enhanced photic driving, enhanced visual stimulation, and a reduced threshold for generating CSD. CSD is a characteristic depolarization wave observed in the migrainous cortex that spreads across the cortex to produce migraine aura [41]. Spontaneous electrical discharges are observed during magnetoencephalography as are a lower threshold and increased incidence of phosphenes after transcranial magnetic stimulation $[42,43]$. A lower threshold for generating phosphenes is observed even with mild head bumps in migraine patients. These patients also show increased continuous negative variation and abnormal habituation in auditory evoked potentials during electrophysiological studies and increased muscle jitter on electromyography. One characteristic of late migraine attacks is cutaneous hypersensitivity (allodynia and hyperpathia). Further, metabolic studies show an increased cerebral metabolic rate of oxygen consumption and glucose use, reduced phosphorylation, and an inability to quickly respond to increased metabolic demand on magnetic spectroscopy [44]. The abnormalities also extend beyond neural tissues and include mitochondrial dysfunctions in platelets and the production of peripheral markers of oxidative stress (nitric oxide metabolites and thiobarbituric acid-reactive species) [41].

The various clinical trials conducted among migraine patients using vitamin $\mathrm{B}_{2}$ are summarized (Table 1). Schoenen et al. (1998) investigated the use of riboflavin $(400 \mathrm{mg} /$ day) among migraine patients $(n=55)$ based on the hypothesis that impaired oxygen metabolism may contribute to the development of migraine attacks [36]. The study reported that approximately $59 \%$ of migraine patients showed at least $50 \%$ of symptom reduction with only minor adverse effects (diarrhea and polyuria) seen. It was also concluded that riboflavin is a good option for migraine prophylaxis due to its high efficacy, excellent tolerability, and low cost. However, in the USA, a randomized, placebo controlled study $(n=48)$ using a combination of high-dose riboflavin $(400 \mathrm{mg} /$ day), magnesium $(300 \mathrm{mg} / \mathrm{day})$, and feverfew $(100 \mathrm{mg} / \mathrm{day})$ found no difference between the high dose $(400 \mathrm{mg} /$ day) of riboflavin and a low dose. Nonetheless, both groups of patients who received either low or high doses of riboflavin had a significant reduction in the number of migraine attacks and the number of days with migraine [45].

A study among Australian children $(n=48)$ reported that the administration of high doses of riboflavin (at least $200 \mathrm{mg} /$ day) did not produce any significant effect in ameliorating migraine when compared to placebo [46]. A study conducted in Italian pediatric and adolescent migraine patients ( $n=41)$, however, suggested that riboflavin (200 or 


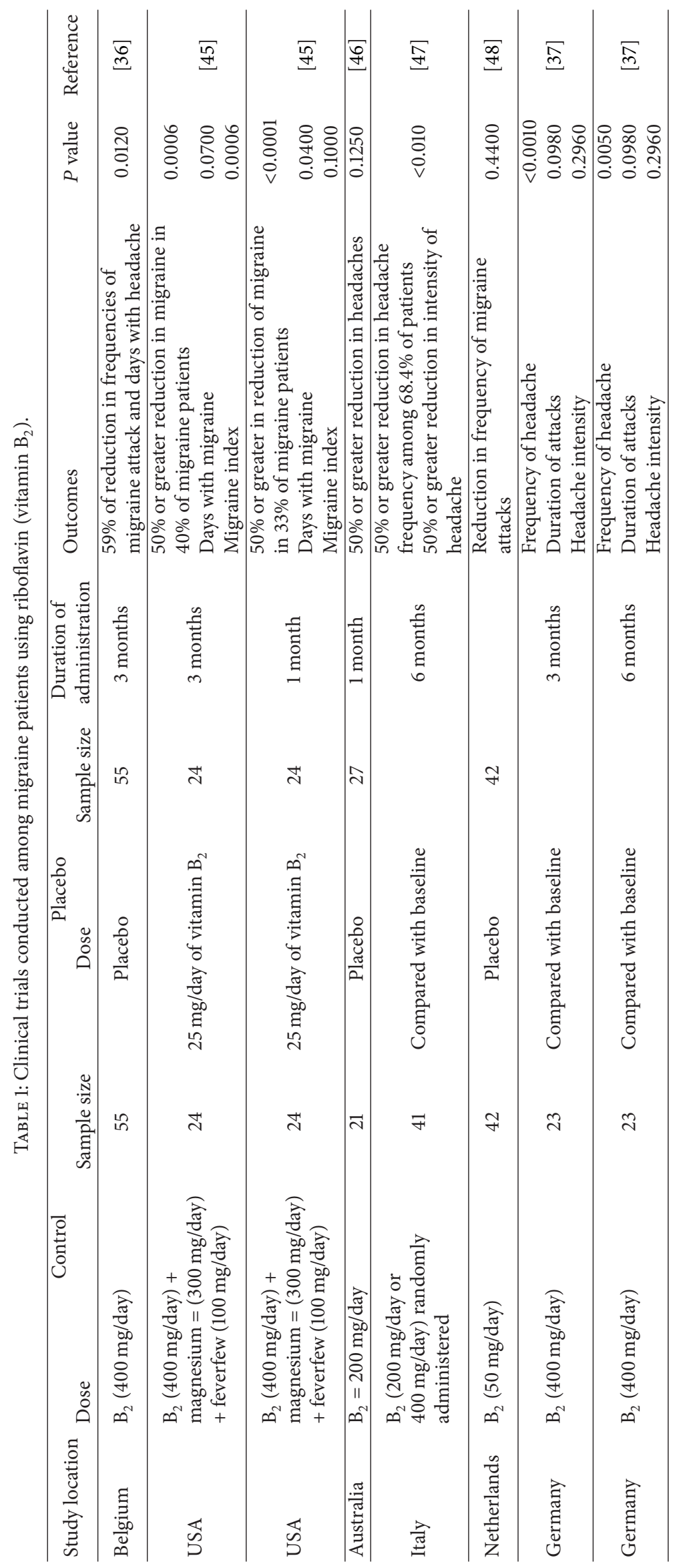


TABLE 2: Characteristics of clinical trials conducted among migraine patients using vitamins $\mathrm{B}_{6}, \mathrm{~B}_{9}$, and $\mathrm{B}_{12}$.

\begin{tabular}{|c|c|c|c|c|c|c|c|}
\hline \multirow{2}{*}{$\begin{array}{l}\text { Study } \\
\text { location }\end{array}$} & \multicolumn{2}{|l|}{ Control } & Placebo & \multirow{2}{*}{$\begin{array}{l}\text { Duration of } \\
\text { administration }\end{array}$} & \multirow{2}{*}{ Outcomes } & \multirow{2}{*}{$P$ value } & \multirow{2}{*}{ Reference } \\
\hline & Dose & $\begin{array}{c}\text { Sample } \\
\text { size }\end{array}$ & Sample size & & & & \\
\hline \multirow{3}{*}{ Australia } & \multirow{3}{*}{$\begin{array}{l}\text { Vitapop tablet containing } \\
\mathrm{B}_{6}(25 \mathrm{mg}), \mathrm{B}_{9}(2 \mathrm{mg}) \& \\
\mathrm{~B}_{12}(400 \mu \mathrm{g}) \text { once daily }\end{array}$} & \multirow{3}{*}{37} & \multirow{3}{*}{ Compared with baseline } & \multirow{3}{*}{6 months } & Migraine disability & 0.010 & \\
\hline & & & & & Frequency of headache & 0.040 & {$[36]$} \\
\hline & & & & & Headache pain severity & 0.002 & \\
\hline \multirow{3}{*}{ Australia } & \multirow{3}{*}{$\begin{array}{l}\text { Vitapop tablet containing } \\
\mathrm{B}_{6}(25 \mathrm{mg}), \mathrm{B}_{9}(2 \mathrm{mg}) \& \\
\mathrm{~B}_{12}(400 \mu \mathrm{g}) \text { once daily }\end{array}$} & \multirow{3}{*}{103} & \multirow{3}{*}{ Compared with baseline } & \multirow{3}{*}{6 months } & Migraine disability & 0.022 & \multirow{3}{*}[45]{} \\
\hline & & & & & Frequency of headache & 0.460 & \\
\hline & & & & & Headache pain severity & 0.017 & \\
\hline
\end{tabular}

$400 \mathrm{mg} /$ day) may be effective in treating migraine [47]. This study suggested that the $200 \mathrm{mg}$ /day dose of riboflavin is sufficient for treating migraine when compared to the $400 \mathrm{mg} / \mathrm{day}$ dose, even though the latter dose is recommended for patients with severe migraine [47]. The study reported that $68.4 \%$ of migraine patients had a $50 \%$ or greater reduction in the frequency of migraine attacks and $21.0 \%$ in its intensity, with only $5 \%$ of patients experiencing minor adverse effects such as vomiting and appetite changes.

A randomized, double blind, placebo controlled, crossover clinical trial of riboflavin conducted among Dutch children ( $n=42 ; 6-13$ years) with migraine administered a medium dose range (approximately $50 \mathrm{mg} /$ day). The study found no significant difference between migraine frequency and the severity or duration of migraine between subjects who received riboflavin or placebo. Therefore, the researchers concluded that there is insufficient evidence for the clinical use of $50 \mathrm{mg}$ of riboflavin as migraine prophylaxis [48]. However, Boehnke et al. (2004) demonstrated the efficacy of riboflavin ( $400 \mathrm{mg} /$ day) among migraine patients $(n=23)$ coming from a tertiary clinic [37]. The study also reported a reduction $(P<0.05)$ in the headache frequency from 4 days/month at baseline to only 2 days/month following riboflavin use but there was no change in either the duration or the intensity of headache experienced.

Due to the conflicting evidence available, more studies, especially randomized clinical trials, are required to conclusively define the exact role of riboflavin as a possible prophylactic agent for migraine. The different race, age, and gender of the subjects involved in various studies add to the variabilities further compounded by the fact that migraine is contributed by multifactorial factors.

2.2. Vitamins $B_{6}, B_{9}$, and $B_{12}$. The flavoenzyme 5,10-methylenetetrahydrofolate reductase (MTHFR) regulates the flow of folate (vitamin $\mathrm{B}_{9}$ ) between the production of nucleotides and the supply of methyl groups during methionine synthesis $[49,50]$ and has major effects on the distribution of intracellular folate [51]. MTHFR also plays a pivotal role in the pathogenesis of migraine and hyperhomocysteinemia. The MTHFR enzyme encoded by MTHFR catalyzes the reduction of 5,10-methylenetetrahydrofolate to 5-methyltetrahydrofolate, an ethyl group donor to the intermediary metabolite homocysteine during its metabolism to methionine. Methionine is used in the synthesis of the universal methyl donor S-adenosylmethionine. Upon donation of the methyl group, S-adenosylmethionine is converted to Sadenosylhomocysteine, which is subsequently hydrolyzed to homocysteine and adenosine [52].

Hyperhomocysteinemia is caused by abnormal methionine biosynthesis due to deficiencies in folate, vitamin $B_{12}$, and vitamin $B_{6}$ [53]. Folate is also needed to drive the methionine synthesis pathway, as a lack of dietary folate and/or reduced MTHFR enzymatic activity can result in increased homocysteine levels in blood plasma [54] (Figure 1). This relationship makes homocysteine a key intermediate in onecarbon metabolism and further clarifies the involvement of B vitamins in transferring one-carbon units and their relation to the plasma concentrations of total homocysteine (Figure 2) $[55,56]$.

Table 2 describes the characteristics of various clinical trials conducted among migraine patients using vitamins $B_{6}$, $\mathrm{B}_{9}$, and $\mathrm{B}_{12}$. A clinical study conducted among Italian children with migraine [32] reported that folic acid ( $5 \mathrm{mg} /$ daily) supplementation for six months led to a significant reduction (52\%) in homocysteine levels during treatment follow-up. However, the study reported no statistically significant effects of folic acid on reducing migraine attacks when the plasma levels of homocysteine were compared at the beginning and end of the treatment.

Lea et al. (2009) reported that vitamin supplementation ( $2 \mathrm{mg}$ of folic acid, $25 \mathrm{mg}$ of vitamin $\mathrm{B}_{6}$, and $400 \mu \mathrm{g}$ of vitamin $\mathrm{B}_{12}$ ) in MA patients reduced homocysteine levels by $39 \%$ when compared to baseline, and the effect was significantly greater than with placebo. Vitamin supplementation also significantly reduced the prevalence of migraine disability from $60 \%$ at baseline to $30 \%$ after 6 months, whereas no reduction was observed in the placebo group. The headache frequency and pain severity were also significantly reduced, with no reductions found in the placebo group. In this patient group, the effect of the treatment on both homocysteine levels and migraine disability was associated with the MTHFR C677T genotype, whereby carriers of the $\mathrm{C}$ allele had a better response to treatment than those with the TT genotype. Therefore, the researchers concluded that lowering homocysteine levels using vitamin supplementation reduces migraine 


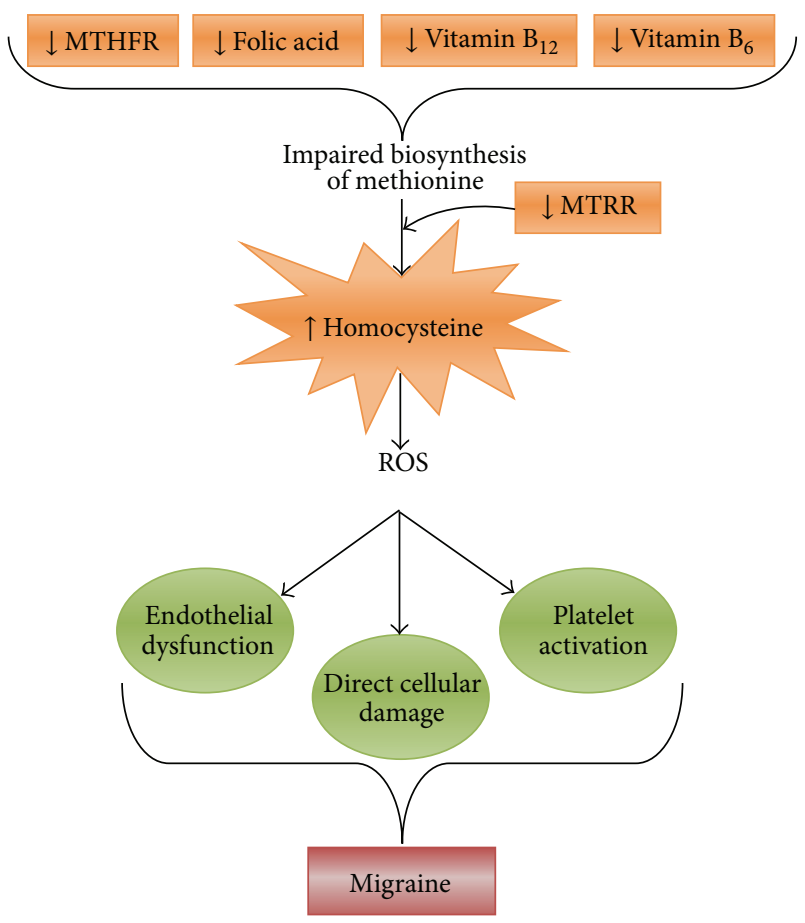

FIGURE 2: Schematic representation depicting the role of vitamins $\mathrm{B}_{6}, \mathrm{~B}_{12}$, and folic acid in migraine pathophysiology. MTRR (or MSR): methionine synthase reductase, MTHFR: methylene tetrahydrofolate reductase, and ROS: reactive oxygen species.

disability in a subgroup of migraine patients [31], particularly in patients with MA.

A study conducted on Australian female Caucasians with MA [35] also reported that vitamin $\left(\mathrm{B}_{6}, \mathrm{~B}_{9}\right.$, and $\left.\mathrm{B}_{12}\right)$ supplementation not only significantly reduced homocysteine levels but also reduced the severity of migraine headache and disability among migraineurs when compared to placebo. When the vitamin-treated group was stratified based on genotype, carriers of the $\mathrm{C}$ allele of the MTHFR C677T variant showed significantly greater reductions in homocysteine levels, the severity of migraine pain, and the percentage of disability when compared with those with the TT genotype. Similarly, the A allele carriers of the methionine synthase reductase (MTRR A66G) variants also showed significantly greater reductions in homocysteine levels, the severity of pain in migraine, and the percentage of migraine disability when compared with those with the GG genotypes. However, despite the genotypic influences of homocysteine levels, the therapeutic efficacy of vitamins $B_{6}, B_{9}$, and $B_{12}$ in patients with the MTRR variant was found to be independent of the $M T H F R$ variant. Further studies are required to elucidate the exact mechanism of interaction between the genetic variants when vitamins are given as prophylaxis to MA patients.

2.3. Vitamin E. Numerous mechanisms have been proposed for menstrual migraine, including a reduction in magnesium levels, platelet dysfunction, central serotonin dysmodulation, and prostaglandin (PG) release $[57,58]$. For example, it has been reported that there is a threefold increase in PG levels in the endometrium between the follicular and luteal phases, which increases further during menstruation (Figure 3). The high levels of PG found in the serum during the premenstrual phase led to the use of drugs with anti-PG effects [59]. Vitamin E inhibits the release of arachidonic acid and the conversion of arachidonic acid to PG by acting on the enzymes phospholipase A2 and cyclooxygenase [60].

Vitamin E, which is an anti-PG agent with a reportedly low side effect profile, can effectively relieve headache pain and associated migraine symptoms [61, 62]. It also reduced functional disability and the need for rescue medications [61, 62]. A limitation of current treatment strategies is that even though a short-term use of prophylactic agents such as nonsteroidal anti-inflammatory drugs and triptans can ameliorate headaches, many patients tend to experience worsening of headache symptoms after the short-term treatment is stopped. However, with the use of vitamin E therapy ( $400 \mathrm{IU} /$ daily for five days during menstruation for 3 cycles), there have been no reported breakthrough headaches. Vitamin E may provide a useful treatment option for women suffering from this debilitating and refractory migraine subtype $[61,62]$. Ziaei et al. reported that there was a reduction in the pain severity and improvement in the functional disability scales among female migraine patients who used vitamin $\mathrm{E}$ for five days during their menstruation periods [61].

Therefore, further studies conducted in a large population could help in determining the efficacy of vitamin $\mathrm{E}$ in the treatment of women who consistently experience menstrual migraine.

2.4. Vitamin C. Epidemiological studies have established an increased risk of developing complex regional pain syndrome (CRPS, a painful neuroinflammatory disorder of the limbs due to tissue or nerve damage) among migraineurs and patients with asthma and inflammatory bowel disease [63]. The link between these seemingly disparate medical conditions may be a shared pathophysiology involving neurogenic inflammation, in which the release of neuropeptides such as substance $\mathrm{P}(\mathrm{SP})$ and calcitonin gene-related peptide (CGRP) produces reactive oxygen species (ROS) that in turn induce tissue damage and microvascular dysfunction [64]. In support of this theory, increased levels of SP, CGRP, and ROS have been found in both CRPS and migraine patients $[65,66]$. Administration of vitamin $\mathrm{C}$, which is a ROS scavenger and is an antioxidant, in doses of $200-1,500 \mathrm{mg}$ daily for up to 50 days following wrist and ankle injuries was shown to significantly reduce the incidence of CRPS and has been proposed as a preventive therapy for this condition [67-71]. It is thought that vitamin C "mops up" ROS produced during neurogenic inflammation in the early stages of CRPS. Based on this model, it seems logical to assume that vitamin C may also modulate the effects of neurogenic inflammation and ROS in migraine.

To date, the efficacy of vitamin $\mathrm{C}$ as a prophylactic treatment for migraine has not been investigated in a randomized controlled trial (RCT). A small nonrandomized trial using 


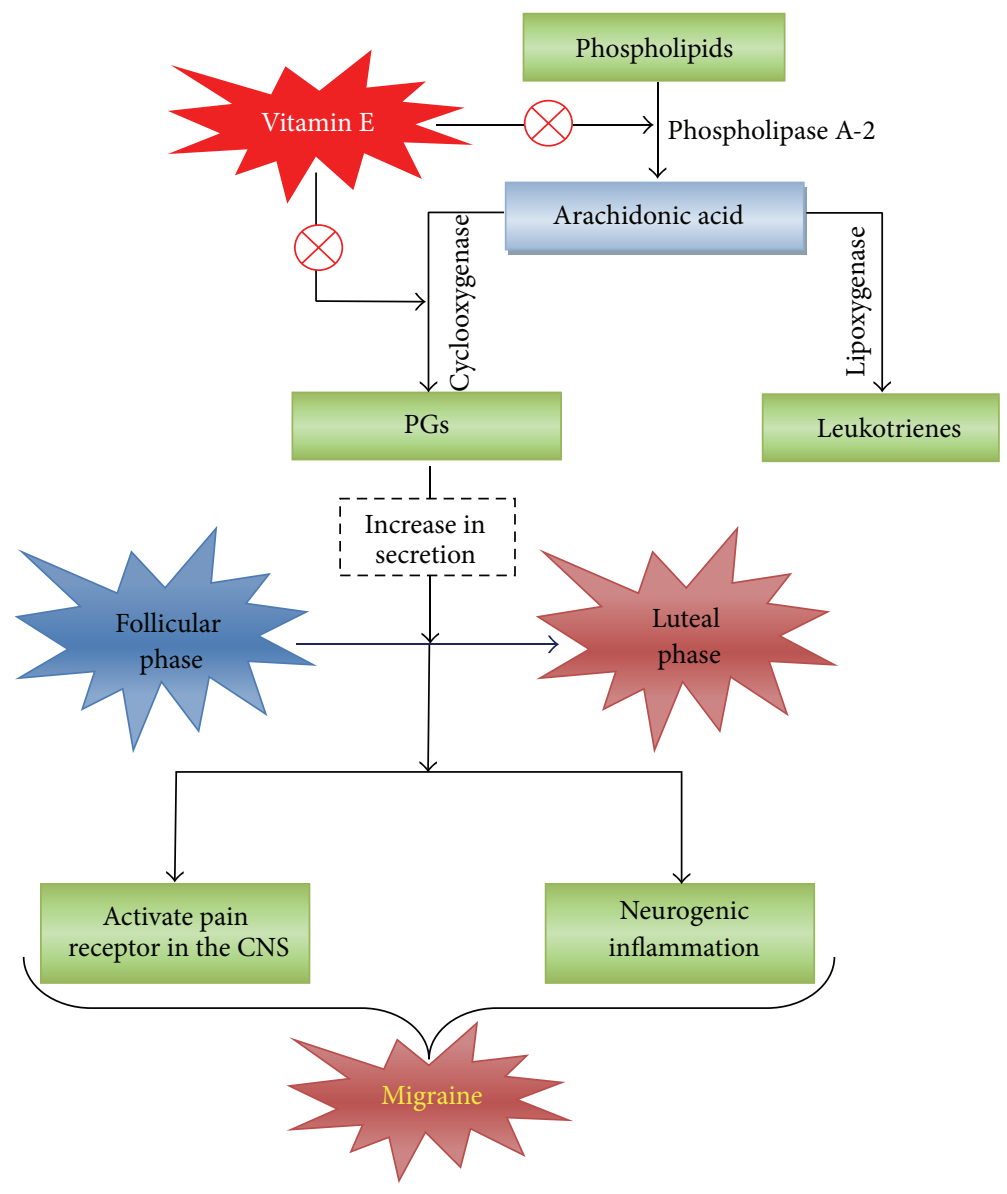

FIGURE 3: Schematic representation of the possible roles of vitamin E in relation to PGs as prophylaxis of menstrual migraine. CNS: central nervous system and PG: prostaglandins. Red circled times symbol: inhibition of the pathway.

a combination of antioxidants and an RCT using the antioxidant vitamin E reported significantly improved migraine outcomes [61,72]. In conclusion, migraine may be considered a "CRPS of the brain," with neurogenic inflammation and ROS generation as common mechanisms. Because vitamin C significantly reduces the risk of CRPS after injury, it may also act as a prophylactic agent against migraine. An RCT investigating the hypothesis that the administration of $1,000 \mathrm{mg}$ of vitamin $\mathrm{C}$ daily will reduce the incidence and severity of migraine attacks is urgently needed, as vitamin $C$ is a cheaper alternative and has relatively fewer side effects than other drugs used for migraine treatment.

\section{Conclusion}

Vitamins are useful for migraine prevention, and riboflavin is reported to be an effective alternative prophylactic agent among pediatric and adult migraine patients by increasing the synthesis of FMN and FAD to generate phosphorylation potentials. Lowering homocysteine levels through vitamin supplementation, specifically with folic acid and vitamins $\mathrm{B}_{6}$ and $\mathrm{B}_{12}$, may reduce migraine disability in patients with MA. However, a larger clinical trial is warranted to establish whether vitamin supplementation using folic acid is a safe, inexpensive, and effective preventative treatment for increasing the quality of life of migraineurs and to determine whether such treatment should be based on the MTHFR genotype. Although vitamin $\mathrm{E}$ has the potential to be used in the treatment of women suffering from menstrual migraine, a debilitating and refractory migraine subtype, further large population studies are needed to determine its efficacy. Additionally, the potential of vitamin $\mathrm{C}$ to be used as an ROS scavenger for neurogenic inflammation in migraine patients needs to be confirmed in RCTs.

\section{Abbreviations}

CGRP: Calcitonin gene-related peptide

CRPS: Complex regional pain syndrome

CSD: Cortical spreading depression

FAD: $\quad$ Flavin adenine dinucleotide

FMN: Flavin mononucleotide

MTHFR: 5,10-Methylenetetrahydrofolate reductase

MTRR: Methionine synthase reductase

MA: $\quad$ Migraine with aura

MO: $\quad$ Migraine without aura

PG: $\quad$ Prostaglandins

QOL: Quality of life 
RCT: Randomized controlled trials

ROS: Reactive oxygen species

SP: Substance P.

\section{Conflict of Interests}

The authors declare that they have no conflict of interests regarding the publication of this paper.

\section{Acknowledgments}

This study was supported by the Universiti Sains Malaysia Research University (RU) Grant (1001/PPSP/815094). The first author was supported by the Graduate Assistance Scheme, School of Medical Science, Universiti Sains Malaysia.

\section{References}

[1] IHS, Internation Headache Society Classification-Migraine, 2004, http://ihs-classification.org/en/02_klassifikation/02_teill/ 01.00.00_migraine.html.

[2] L. E. Morillo, "Migraine headache," Clinical Evidence, no. 11, pp. 1696-1719, 2004.

[3] R. B. Lipton, M. E. Bigal, M. Diamond, F. Freitag, M. L. Reed, and W. F. Stewart, "Migraine prevalence, disease burden, and the need for preventive therapy," Neurology, vol. 68, no. 5, pp. 343-349, 2007.

[4] "Classification and diagnostic criteria for headache disorders, cranial neuralgias and facial pain. Headache Classification Committee of the International Headache Society," Cephalalgia, vol. 8, supplement 7, pp. 1-96, 1988.

[5] IHS Classification ICHD II-Migraine -1-G43, 2004, http:// ihs-classification.org/en/02_klassifikation/02_teill/01.00.00_migraine.html.

[6] World Health Organisation, Headache disorders. Fact Sheet no. 277, 2012, http://www.who.int/mediacentre/factsheets/fs277/ en/index.html.

[7] M. Sparaco, M. Feleppa, R. B. Lipton, A. M. Rapoport, and M. E. Bigal, "Mitochondrial dysfunction and migraine: evidence and hypotheses," Cephalalgia, vol. 26, no. 4, pp. 361-372, 2006.

[8] P. Montagna, T. Sacquegna, P. Martinelli et al., "Mitochondrial abnormalities in migraine. Preliminary findings," Headache, vol. 28, no. 7, pp. 477-480, 1988.

[9] S. Stuart and L. R. Griffiths, "A possible role for mitochondrial dysfunction in migraine," Molecular Genetics and Genomics, vol. 287, no. 11-12, pp. 837-844, 2012.

[10] A. M. Schaefer, R. McFarland, E. L. Blakely et al., "Prevalence of mitochondrial DNA disease in adults," Annals of Neurology, vol. 63, no. 1, pp. 35-39, 2008.

[11] H. K. Kim, S. K. Park, J.-L. Zhou et al., "Reactive oxygen species (ROS) play an important role in a rat model of neuropathic pain," Pain, vol. 111, no. 1-2, pp. 116-124, 2004.

[12] M. R. Metea and E. A. Newman, "Glial cells dilate and constrict blood vessels: a mechanism of neurovascular coupling," The Journal of Neuroscience, vol. 26, no. 11, pp. 2862-2870, 2006.

[13] A. A. Leao, "Further observations on the spreading depression of activity in the cerebral cortex," Journal of Neurophysiology, vol. 10, no. 6, pp. 409-414, 1947.
[14] T. Shimomura, A. Kitano, H. Marukawa et al., "Point mutation in platelet mitochondrial tRNA(Leu(UUR)) in patient with cluster headache," The Lancet, vol. 344, no. 8922, p. 625, 1994.

[15] E. A. Zaki, T. Freilinger, T. Klopstock et al., “Two common mitochondrial DNA polymorphisms are highly associated with migraine headache and cyclic vomiting syndrome," Cephalalgia, vol. 29, no. 7, pp. 719-728, 2009.

[16] R. Gupta, R. Pathak, M. S. Bhatia, and B. D. Banerjee, "Comparison of oxidative stress among migraineurs, tension-type headache subjects, and a control group," Annals of Indian Academy of Neurology, vol. 12, no. 3, pp. 167-172, 2009.

[17] D. Tuncel, F. I. Tolun, M. Gokce, S. Imrek, and H. Ekerbiçer, "Oxidative stress in migraine with and without aura," Biological Trace Element Research, vol. 126, no. 1-3, pp. 92-97, 2008.

[18] I. Ciancarelli, M. G. Tozzi-Ciancarelli, C. Di Massimo, C. Marini, and A. Carolei, "Urinary nitric oxide metabolites and lipid peroxidation by-products in migraine," Cephalalgia, vol. 23, no. 1, pp. 39-42, 2003.

[19] X. H. Hu, L. E. Markson, R. B. Lipton, W. F. Stewart, and M. L. Berger, "Burden of migraine in the United States: disability and economic costs," Archives of Internal Medicine, vol. 159, no. 8, pp. 813-818, 1999.

[20] S. D. Silberstein and W. Edlund, "Practice parameter: evidencebased guidelines for migraine headache (an evidence-based review): report of the Quality Standards Subcommittee of the American Academy of Neurology," Neurology, vol. 55, no. 6, pp. 754-762, 2000.

[21] S. Evers, J. Áfra, A. Frese et al., "EFNS guideline on the drug treatment of migraine-revised report of an EFNS task force," European Journal of Neurology, vol. 16, no. 9, pp. 968-981, 2009.

[22] F. Antonaci, C. Dumitrache, I. De Cillis, and M. Allena, "A review of current European treatment guidelines for migraine," The Journal of Headache and Pain, vol. 11, no. 1, pp. 13-19, 2010.

[23] K. Linde and K. Rossnagel, "Propranolol for migraine prophylaxis," Cochrane Database of Systematic Reviews, no. 2, Article ID CD003225, 2004.

[24] L. Reveiz-Herault, A. F. Cardona, E. G. Ospina, and P. Carrillo, "Effectiveness of flunarizine in the prophylaxis of migraine: a meta-analytical review of the literature," Revista de Neurología, vol. 36, no. 10, pp. 907-912, 2003.

[25] M. Linde, W. M. Mulleners, E. P. Chronicle, and D. C. McCrory, "Topiramate for the prophylaxis of episodic migraine in adults," The Cochrane Database of Systematic Reviews, vol. 6, Article ID CD010610, 2013.

[26] P. L. Moja, C. Cusi, R. R. Sterzi, and C. Canepari, "Selective serotonin re-uptake inhibitors (SSRIs) for preventing migraine and tension-type headaches," Cochrane Database of Systematic Reviews, no. 3, Article ID CD002919, 2005.

[27] E. Chronicle and W. Mulleners, "Anticonvulsant drugs for migraine prophylaxis," Cochrane Database of Systematic Reviews, no. 3, Article ID CD003226, 2004.

[28] S. L. Orr and S. Venkateswaran, "Nutraceuticals in the prophylaxis of pediatric migraine: evidence-based review and recommendations," Cephalalgia, vol. 34, no. 8, pp. 568-583, 2014.

[29] P. S. Sândor, L. Di Clemente, G. Coppola et al., "Efficacy of coenzyme Q10 in migraine prophylaxis: a randomized controlled trial," Neurology, vol. 64, no. 4, pp. 713-715, 2005.

[30] P. Schiapparelli, G. Allais, I. C. Gabellari, S. Rolando, M. G. Terzi, and C. Benedetto, "Non-pharmacological approach to migraine prophylaxis: part II," Neurological Sciences, vol. 31, supplement 1, pp. S137-S139, 2010. 
[31] R. Lea, N. Colson, S. Quinlan, J. MacMillan, and L. Griffiths, "The effects of vitamin supplementation and MTHFR (C677T) genotype on homocysteine-lowering and migraine disability," Pharmacogenetics and Genomics, vol. 19, no. 6, pp. 422-428, 2009.

[32] G. Di Rosa, S. Attinà, M. Spanò et al., "Efficacy of folic acid in children with migraine, hyperhomocysteinemia and MTHFR polymorphisms," Headache, vol. 47, no. 9, pp. 1342-1344, 2007.

[33] T. Dalsgaard-Nielsen and J. Trautmann, "Prophylactic treatment of migraine with vitamin B 12," Ugeskrift for Laeger, vol. 132, no. 7, pp. 339-341, 1970.

[34] A. Bianchi, S. Salomone, F. Caraci, V. Pizza, R. Bernardini, and C. C. D’Amato, "Role of magnesium, coenzyme Q10, riboflavin, and vitamin B12 in migraine prophylaxis," Vitamins and Hormones, vol. 69, pp. 297-312, 2004.

[35] S. Menon, R. A. Lea, B. Roy et al., "Genotypes of the MTHFR C677T and MTRR A66G genes act independently to reduce migraine disability in response to vitamin supplementation," Pharmacogenetics and Genomics, vol. 22, no. 10, pp. 741-749, 2012.

[36] J. Schoenen, J. Jacquy, and M. Lenaerts, "Effectiveness of high-dose riboflavin in migraine prophylaxis: a randomized controlled trial," Neurology, vol. 50, no. 2, pp. 466-470, 1998.

[37] C. Boehnke, U. Reuter, U. Flach, S. Schuh-Hofer, K. M. Einhäupl, and G. Arnold, "High-dose riboflavin treatment is efficacious in migraine prophylaxis: an open study in a tertiary care centre," European Journal of Neurology, vol. 11, no. 7, pp. 475-477, 2004.

[38] B. Barbiroli, P. Montagna, P. Cortelli et al., "Abnormal brain and muscle energy metabolism shown by 31P magnetic resonance spectroscopy in patients affected by migraine with aura," Neurology, vol. 42, no. 6, pp. 1209-1214, 1992.

[39] P. Montagna, P. Cortelli, L. Monari et al., "31P-Magnetic resonance spectroscopy in migraine without aura," Neurology, vol. 44, no. 4, pp. 666-669, 1994.

[40] W. F. M. Arts, H. R. Scholte, J. M. Bogaard, K. F. Kerrebijn, and I. E. M. Luyt-Houwen, "NADH-CoQ reductase deficient myopathy: successful treatment with riboflavin," The Lancet, vol. 2, no. 8349, pp. 581-582, 1983.

[41] N. M. Ramadan, "Prophylactic migraine therapy: mechanisms and evidence," Current Pain and Headache Reports, vol. 8, no. 2, pp. 91-95, 2004.

[42] G. L. Barkley, N. Tepley, S. Nagel-Leiby, J. E. Moran, R. T. Simkins, and K. M. A. Welch, "Magnetoencephalographic studies of migraine," Headache, vol. 30, no. 7, pp. 428-434, 1990.

[43] S. K. Aurora and F. Wilkinson, "The brain is hyperexcitable in migraine," Cephalalgia, vol. 27, no. 12, pp. 1442-1453, 2007.

[44] K. M. A. Welch, S. R. Levine, G. D’Andrea, L. R. Schultz, and J. A. Helpern, "Preliminary observations on brain energy metabolism in migraine studied by in vivo phosphorus 31 NMR spectroscopy," Neurology, vol. 39, no. 4, pp. 538-541, 1989.

[45] M. Maizels, A. Blumenfeld, and R. Burchette, "A combination of riboflavin, magnesium, and feverfew for migraine prophylaxis: a randomized trial," Headache, vol. 44, no. 9, pp. 885-890, 2004.

[46] S. C. MacLennan, F. M. Wade, K. M. L. Forrest, P. D. Ratanayake, E. Fagan, and J. Antony, "High-Dose ribof lavin for migraine prophylaxis in children: a double-blind, randomized, placebocontrolled trial," Journal of Child Neurology, vol. 23, no. 11, pp. 1300-1304, 2008.

[47] M. Condó, A. Posar, A. Arbizzani, and A. Parmeggiani, "Riboflavin prophylaxis in pediatric and adolescent migraine,"
The Journal of Headache and Pain, vol. 10, no. 5, pp. 361-365, 2009.

[48] J. Bruijn, H. Duivenvoorden, J. Passchier, H. Locher, N. Dijkstra, and W.-F. Arts, "Medium-dose riboflavin as a prophylactic agent in children with migraine: a preliminary placebocontrolled, randomised, double-blind, cross-over trial," Cephalalgia, vol. 30, no. 12, pp. 1426-1434, 2010.

[49] C. Kutzbach and E. L. R. Stokstad, "Mammalian methylenetetrahydrofolate reductase Partial purification, properties, and inhibition by S-adenosylmethionine," Biochimica et Biophysica Acta, vol. 250, no. 3, pp. 459-477, 1971.

[50] J. Selhub, "Homocysteine metabolism," Annual Review of Nutrition, vol. 19, pp. 217-246, 1999.

[51] P. J. Bagley and J. Selhub, "A common mutation in the methylenetetrahydrofolate reductase gene is associated with an accumulation of formylated tetrahydrofolates in red blood cells," Proceedings of the National Academy of Sciences of the United States of America, vol. 95, no. 22, pp. 13217-13220, 1998.

[52] H. Jakubowski, "Pathophysiological consequences of homocysteine excess," The Journal of Nutrition, vol. 136, no. 6, supplement, pp. 1741S-1749S, 2006.

[53] K. S. McCully, "Homocysteine, vitamins, and vascular disease prevention," The American Journal of Clinical Nutrition, vol. 86, no. 5, pp. 1563S-1568S, 2007.

[54] I. M. Graham and P. O'Callaghan, "Vitamins, homocysteine and cardiovascular risk," Cardiovascular Drugs and Therapy, vol. 16, no. 5, pp. 383-389, 2002.

[55] S. E. Vollset, H. Refsum, and P. M. Ueland, "Population determinants of homocysteine," The American Journal of Clinical Nutrition, vol. 73, no. 3, pp. 499-500, 2001.

[56] M. M. Shaik, T. H. Lin, M. A. Kamal, and S. H. Gan, "Do folate, vitamins $B_{6}$ and $B_{12}$ play a role in the pathogenesis of migraine? The role of pharmacoepigenomics," CNS \& Neurological Disorders-Drug Targets, vol. 13, no. 5, pp. 828-835, 2014.

[57] E. A. MacGregor, "Menstrual' migraine: towards a definition," Cephalalgia, vol. 16, no. 1, pp. 11-21, 1996.

[58] R. Salonen and J. Saiers, "Sumatriptan is effective in the treatment of menstrual migraine: a review of prospective studies and retrospective analyses," Cephalalgia, vol. 19, no. 1, pp. 16-19, 1999.

[59] F. Granella, G. Sances, G. Messa, M. de Marinis, and G. C. Manzoni, "Treatment of menstrual migraine," Cephalalgia, Supplement, vol. 17, supplement 20, pp. 35-38, 1997.

[60] S. Ziaei, M. Zakeri, and A. Kazemnejad, "A randomised controlled trial of vitamin $\mathrm{E}$ in the treatment of primary dysmenorrhoea," British Journal of Obstetrics and Gynaecology, vol. 112, no. 4, pp. 466-469, 2005.

[61] S. Ziaei, A. Kazemnejad, and A. Sedighi, "The effect of vitamin E on the treatment of menstrual migraine," Medical Science Monitor, vol. 15, no. 1, pp. CR16-CR19, 2009.

[62] E. A. MacGregor, "Prevention and treatment of menstrual migraine," Drugs, vol. 70, no. 14, pp. 1799-1818, 2010.

[63] M. de Mos, F. J. P. M. Huygen, J. P. Dieleman, J. S. H. A. Koopman, B. H. C. Stricker, and M. C. J. M. Sturkenboom, "Medical history and the onset of complex regional pain syndrome (CRPS)," Pain, vol. 139, no. 2, pp. 458-466, 2008.

[64] A. Starr, R. Graepel, J. Keeble et al., "A reactive oxygen speciesmediated component in neurogenic vasodilatation," Cardiovascular Research, vol. 78, no. 1, pp. 139-147, 2008.

[65] E. Fusayasu, H. Kowa, T. Takeshima, K. Nakaso, and K. Nakashima, "Increased plasma substance P and CGRP levels, 
and high ACE activity in migraineurs during headache-free periods," Pain, vol. 128, no. 3, pp. 209-214, 2007.

[66] T. Wei, W.-W. Li, T.-Z. Guo et al., "Post-junctional facilitation of Substance $\mathrm{P}$ signaling in a tibia fracture rat model of complex regional pain syndrome type I," Pain, vol. 144, no. 3, pp. 278286, 2009.

[67] S. J. Padayatty, A. Katz, Y. Wang et al., "Vitamin C as an antioxidant: evaluation of its role in disease prevention," Journal of the American College of Nutrition, vol. 22, no. 1, pp. 18-35, 2003.

[68] R. S. Perez, P. E. Zollinger, P. U. Dijkstra et al., "Evidence based guidelines for complex regional pain syndrome type 1," $B M C$ Neurology, vol. 10, article 20, 2010.

[69] P. E. Zollinger, W. E. Tuinebreijer, R. W. Kreis, and R. S. Breederveld, "Effect of vitamin C on frequency of reflex sympathetic dystrophy in wrist fractures: a randomised trial," The Lancet, vol. 354, no. 9195, pp. 2025-2028, 1999.

[70] P. E. Zollinger, W. E. Tuinebreijer, R. S. Breederveld, and R. W. Kreis, "Can vitamin C prevent complex regional pain syndrome in patients with wrist fractures? A randomized, controlled, multicenter dose-response study," The Journal of Bone and Joint Surgery-American Volume, vol. 89, no. 7, pp. 1424-1431, 2007.

[71] J.-L. Besse, S. Gadeyne, S. Galand-Desmé, J.-L. Lerat, and B. Moyen, "Effect of vitamin $\mathrm{C}$ on prevention of complex regional pain syndrome type I in foot and ankle surgery," Foot and Ankle Surgery, vol. 15, no. 4, pp. 179-182, 2009.

[72] S. Chayasirisobhon, "Use of a pine bark extract and antioxidant vitamin combination product as therapy for migraine in patients refractory to pharmacologic medication," Headache, vol. 46, no. 5, pp. 788-793, 2006. 

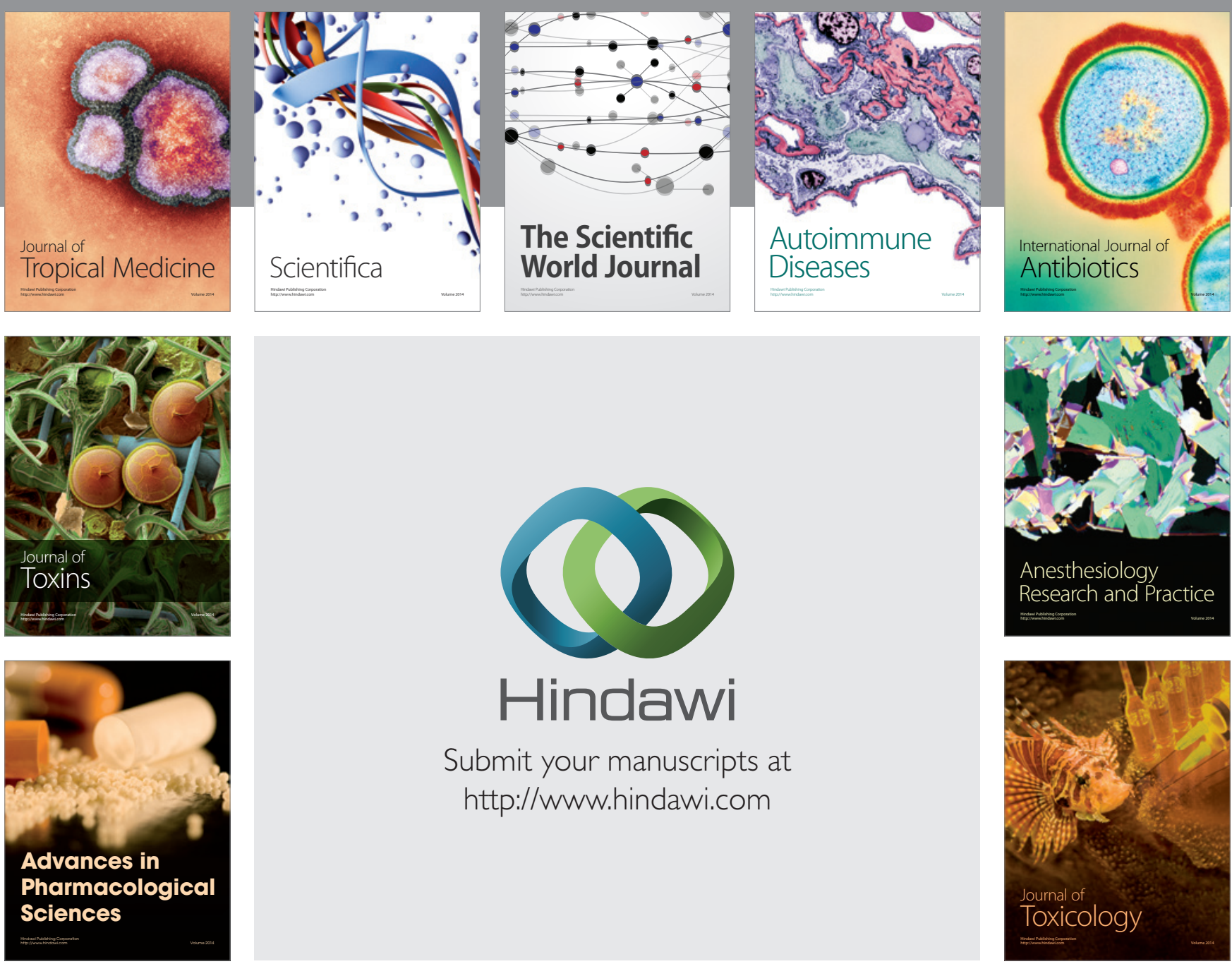

\section{Hindawi}

Submit your manuscripts at

http://www.hindawi.com
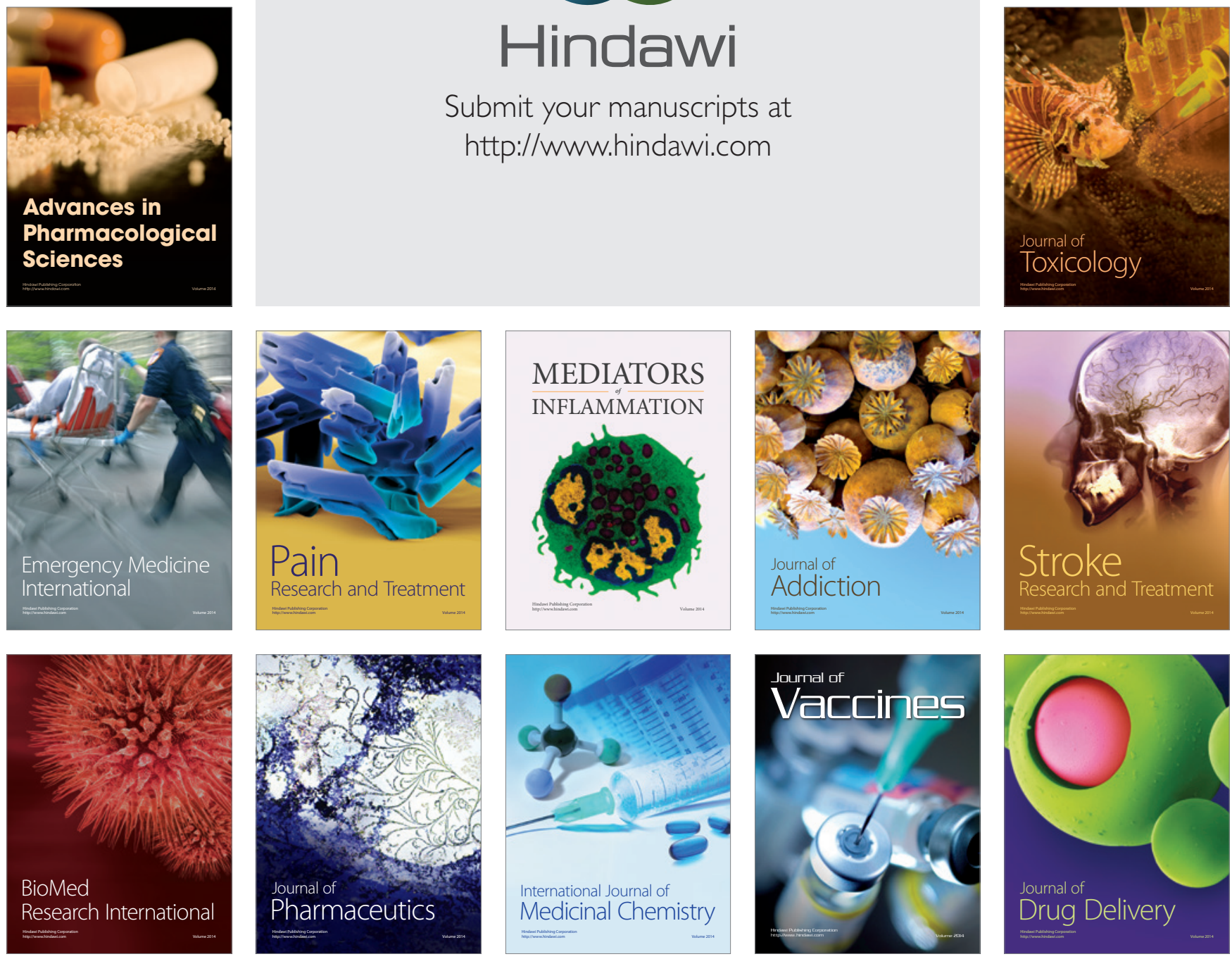